\title{
Erratum: Generalizing the relativistic quantization condition to include all three-pion isospin channels
}

\author{
Maxwell T. Hansen, ${ }^{a}$ Fernando Romero-López ${ }^{b}$ and Stephen R. Sharpe ${ }^{c}$ \\ a Theoretical Physics Department, CERN, \\ Geneva 23 1211, Switzerland \\ ${ }^{b}$ IFIC, CSIC-Universitat de València, \\ Paterna 46980, Spain \\ ${ }^{c}$ Physics Department, University of Washington, \\ Seattle, WA 98195-1560, U.S.A. \\ E-mail: maxwell.hansen@cern.ch, fernando.romero@uv.es, srsharpe@uw.edu
}

ERRATUM TO: JHEP07(2020)047

ARXIV EPRINT: 2003.10974

We have found an error in a statement following eq. (2.5) of our paper, concerning the function $f(a, b, k)$ that first appears in that equation. The error does not affect the main results of the published manuscript but leads to various erroneous statements about auxiliary quantities used in the derivation, specifically the matrices $\sigma(k, a), \sigma^{\dagger}(k, a)$ as well as the endcap factors $A_{3}$ and $A_{3}^{\prime}$.

The issue arises in the statement that it is convenient to take the function $f(a, b, k)$ to be exchange symmetric with respect to its three arguments. This has the unwanted consequence of making six of the seven operators in the column vector of eq. (2.4) identically equal. This, in turn, implies that many operators are identically zero in the definite isospin basis, considered in section 2.4. To repair this, the last sentence of the paragraph containing eq. (2.4), starting "It is convenient for the subsequent...", should be removed, as should footnote 3 and the next paragraph, beginning with "At this point, the reader may wonder why...". The deleted text should be replaced with the following:

As we discuss further below, it is crucial that $f(a, b, k)$ is not symmetric with respect to permutations of its arguments. More precisely, $f(a, b, k)$ is defined such that all seven operators defining $\mathcal{O}_{j}(x)$ are in fact distinct, which is necessary to ensure that all definite-isospin operators are non-zero. 
The next modification begins with the sentence "The exact relation is $\sigma_{j l}(k, a)=$ $M_{j l} f(a, b, k)$, where" appearing immediately before eq. (2.24). The text and also eq. (2.24) should be removed and replaced with the following:

The exact relation is

$$
\sigma(k, a)=\left(\begin{array}{ccccccc}
f(a, b, k) & f(b, a, k) & f(a, k, b) & 0 & f(b, k, a) & f(k, a, b) & f(k, b, a) \\
f(b, a, k) & f(a, b, k) & f(k, a, b) & 0 & f(k, b, a) & f(a, k, b) & f(b, k, a) \\
f(a, k, b) & f(b, k, a) & f(a, b, k) & 0 & f(b, a, k) & f(k, b, a) & f(k, a, b) \\
0 & 0 & 0 & g(a, b, k) & 0 & 0 & 0 \\
f(k, a, b) & f(k, b, a) & f(b, a, k) & 0 & f(a, b, k) & f(b, k, a) & f(a, k, b) \\
f(b, k, a) & f(a, k, b) & f(k, b, a) & 0 & f(k, a, b) & f(a, b, k) & f(b, a, k) \\
f(k, b, a) & f(k, a, b) & f(b, k, a) & 0 & f(a, k, b) & f(b, a, k) & f(a, b, k)
\end{array}\right),
$$

where

$$
g(a, b, k) \equiv f(a, b, k)+f(b, a, k)+f(a, k, b)+f(b, k, a)+f(k, a, b)+f(k, b, a)
$$

is the symmetrized version of $f(a, b, k)$. Here the $(i, j)$ entry of the matrix can be understood as the non-interacting overlap of the operator $\mathcal{O}_{i}(0)$ with the $j$ th state. The latter is defined with the convention of eq. (2.4). So, for example, the $(1,3)$ entry follows from

$$
\begin{aligned}
\left\langle 0\left|\mathcal{O}_{1}(0)\right| \pi \pi \pi, j=3\right\rangle & =\int_{a^{\prime}, b^{\prime}, k^{\prime}}\left\langle 0\left|f\left(a^{\prime}, b^{\prime}, k^{\prime}\right) \widetilde{\pi}_{-}\left(a^{\prime}\right) \widetilde{\pi}_{0}\left(b^{\prime}\right) \widetilde{\pi}_{+}\left(k^{\prime}\right)\right| \pi_{-}(a) \pi_{+}(b) \pi_{0}(k)\right\rangle, \\
& =f(a, k, b),
\end{aligned}
$$

where $|\pi \pi \pi, j=3\rangle$ represents the non-interacting state with momentum assignment given by the index. The 6 different terms in the $(4,4)$ entry arise from the 3 ! contractions of the neutral operator with the neutral state.

In these adjustments the quantity $M$ is no longer needed and is replaced everywhere with $\sigma_{i j}(k, a)$. In addition the parenthetical remark: "(Here and below we use empty and filled squares to present matrices of 0 s and $1 \mathrm{~s}$ as we find this form more readable.)" is now first relevant after eq. (2.38) and should be moved to this location.

Finally, in the paragraph preceding eq. (2.25), the discussion of the factor of 6 should be modified. Starting with the sentence "Second, if both $j, l \neq 4$ then one recovers a nonzero contribution with a factor of...", the remainder of the paragraph should be replaced with the following:

Second, if both $j, l \neq 4$ then one recovers a non-zero contribution with a factor of 6 arising from the contracted matrix indices. For example for $j=1, l=2$ one finds

$$
\begin{aligned}
\sum_{k} \sigma_{1 k}(k, a) \sigma_{k 2}^{\dagger}(k, a)= & f(a, b, k) f^{*}(b, a, k)+f(b, a, k) f^{*}(a, b, k)+f(a, k, b) f^{*}(k, a, b) \\
& +f(b, k, a) f^{*}(k, b, a)+f(k, a, b) f^{*}(a, k, b)+f(k, b, a) f^{*}(b, k, a) \\
\rightarrow & 6 f(a, b, k) f^{*}(b, a, k),
\end{aligned}
$$


where the arrow represents a replacement in the integral that is justified as all other factors are exchange symmetric with respect to $a, b$, and $k$. This compensates the $1 / 6$ pre-factor, leading to the correct expression for a diagram with three distinguishable particles. Finally, $j=l=4$ yields the diagram with three neutral particles and in this case the $1 / 6$ survives and correctly gives the symmetry factor for identical particles.

These adjustments complete the redefinitions of $\sigma(k, a)$ and $\sigma^{\dagger}(k, a)$ and the adjustments to $A_{3}$ and $A_{3}^{\prime}$ follow automatically. All remaining equations and, in particular, all the main results and conclusions of the paper are unchanged. The implicit assumption that the on-diagonal entries of $A_{3}$ and $A_{3}^{\prime}$ are non-zero in the isospin basis, introduced in section 2.4, is now correct.

For completeness, we also note an additional minor correction. The sum following eq. (2.5) should be defined with an implicit factor of $1 / L^{3}$, but this is never stated explicitly. To correct this, the following should be inserted after the sentence containing eq. (2.5): "We also adopt the convention here, and below, that the factor of $1 / L^{3}$ accompanying each sum is left implicit".

Open Access. This article is distributed under the terms of the Creative Commons Attribution License (CC-BY 4.0), which permits any use, distribution and reproduction in any medium, provided the original author(s) and source are credited. 